Transactions of the 13th International Conference on Structural Mechanics in Reactor Technology (SMiRT 13), Escola de Engenharia - Universidade Federal do Rio Grande do Sul, Porto Alegre, Brazil, August 13-18, 1995

\title{
Simulation of isothermal fission gas release
}

Denis, A., Piotrkowski, $\mathbf{R}$.

Comisión Nacional de Energia Atómica, Depto. Combustibles Nucleares, Buenos Aires, Argentina

\begin{abstract}
A fission gas release model is presented, which considers the atomic diffusion with trapping of $\mathrm{Xe}$ and $\mathrm{Kr}$ atoms generated during irradiation of the $\mathrm{NO}_{2}$ fuel, precipitation of gas in intra and intergranular bubbles, its resolution due to impact of energetic fission fragments, interconnection of grain boundary bubbles and sweeping of gas by the grain boundary movement. The diffusion equation is solved by the finite difference method and the results compare well with isothermal experimental determinations.
\end{abstract}

\section{OUTLINE OF THE MODEL}

The fission gases, mainly $\mathrm{Xe}$ and $\mathrm{Kr}$, produced by irradiated nuclear fuels affect the fuel performance: they diminish the heat transfer between fuel and cladding causing an increase in the rod temperature, and they increase the internal rod pressure which may lead to cladding failure and to the release of fission products to the atmosphere. Due to the virtually complete insolubility in the fuel matrix, the rejected gases either are released from the fuel or they precipitate forming bubbles within the fuel. Irradiation can cause bubbles destruction. A nonzero dynamical solubility of noble gases appears since complete precipitation in bubbles is impossible because of kinetic reasons (Olander 1976).

The $\mathrm{UO}_{2}$ is considered as a collection of spherical grains. The intragranular (intrag.) bubbles of a few $\mathrm{nm}$, are considered immobile, acting as gas atoms traps. Part of the free atoms diffuse to the grain boundaries (g.b.), where they give origin to intergranular (interg.), lenticular bubbles of the order of $\mu \mathrm{m}$. The gas atoms in either inter or intrag. bubbles, can re-enter the matrix due to impact of fission fragments. The amount of gas stored in the interg. bubbles grows up to a saturation value. Thereafter, interg. bubbles interconnect and the gas in excess is released through different channels to the external surface of the fuel. The resolution of interg. bubbles affects mainly the region of the grain adjacent to the $(g \cdot b)$. During grain growth the g.b. traps the 
gas atoms, either free or in intrag. bubbles, contained in the swept volume. The g.b. is considered as a perfect sink (Nakajima et al. 1987). Due to spherical symmetry, the concentration gradient is null at the center of the grain.

The diffusion equation in a grain of radius $a$ is

$$
\frac{\partial c}{\partial t}=D\left(\frac{\partial^{2} c}{\partial r^{2}}+\frac{2}{r} \frac{\partial c}{\partial r}\right)-g c+b m+\beta
$$

where $c$ is the concentration of dissolved gas $\left(\right.$ at $\mathrm{m}^{-3}$ ), $m$ the concentration of gas in intrag. bubbles $\left(\right.$ at $\left.\mathrm{m}^{-3}\right), \beta$ the gas production rate $\left(\right.$ at $\left.\mathrm{m}^{-3} \mathrm{~s}^{-1}\right), D$ the single gas atom diffusion coefficient $\left(\mathrm{m}^{2} \mathrm{~s}^{-9}\right), g$ the probability of capture of a dissolved gas atom by a trap $\left(s^{-1}\right)$ and $b$ the probability of resolution of trapped atoms $\left(s^{-1}\right)$.

The balance equation for the trapped atoms is

$$
\frac{\partial m}{\partial t}=g c-b m
$$

The total concentration of gas within a grain, $P=c+m$, obeys

$$
\frac{\partial \psi}{\partial t}-D\left(\frac{\partial^{2} C}{\partial r^{2}}+\frac{2}{r} \frac{\partial C}{\partial r}\right)+\beta
$$

Under stationary trapping conditions, the amount of trapped gas $m$, remains unchanged and

$$
g c=b m \quad, \quad \psi=c\left(1+\frac{g}{b}\right) \quad \text { and } \quad \frac{\partial \psi}{\partial t}=D^{\prime}\left(\frac{\partial^{2} \psi}{\partial r^{2}}+\frac{2}{r} \frac{\partial \psi}{\partial r}\right)+\beta
$$

where $D^{\prime}=D b /(b+g)$ is the effective diffusion coefficient within a grain (White et al. 1983).

According to Turnbull et al. (1987) the diffusion coefficient is given by $D=D_{1}+4 D_{2}+4 D_{3}$ with

$$
D_{1}=7.6 \times 10^{-10} \exp \left(-7 \times 10^{4} / R T\right) \quad\left[\mathrm{m}^{2} \mathrm{~s}^{-1}\right]
$$

where $R=1.987 \mathrm{cal} \mathrm{mol}^{-1} \mathrm{~K}^{-1}$ and $T$ is the absolute temperature,

$$
D_{2}-S^{2} j_{V} V
$$

$S=\Omega^{1 / 3}$ is the atomic jump distance, $j_{V}=10^{13} \exp \left(-5.52 \times 10^{4} / R T\right) \mathrm{s}^{-1}$ is the vacancy jump frequency and $V$ is the vacancies concentration given by Turnbull et al. (1982), and

$$
D_{3}=2 \times 10^{-40} \mathrm{~F} \quad\left[\mathrm{~m}^{2} \mathrm{~s}^{-1}\right]
$$

The bubbles density $C_{B}$ is given by

$$
C_{B}=\frac{1.52 \alpha}{\pi I_{t}\left(\bar{R}+Z_{0}\right)^{2}}
$$

where $\alpha$ is the number of bubbles nucleated by each fission fragment, $I_{f}$ is the distance a fission fragment travels before loosing its energy, $z_{0}$ is the range of influence of a fission fragment along its path and $\bar{R}$ is the mean radius of bubbles given by 
The capture rate at bubbles, $g$, and the resolution rate, $b$, of trapped gas atoms back into solution (Nakajima 1987) are

$$
g=4 \pi \bar{R} D C_{B} \quad\left[s^{-1}\right] \quad ; b-3.03 F \pi I_{f}\left(R+Z_{0}\right)^{2} \quad\left[s^{-1}\right]
$$

Irradiation is assumed to produce an homogeneous distribution of fissions within the grain at a rate $F$. The overall yield, $y$, of a given nuclide includes the contribution due to beta decay of the precursors in the mass chain in addition to the independent yield of the nuclide. Thus, the uniform gas generation rate is $Y F$.

Bubbles resolution provides an additional source of gas atoms which penetrate into the grain specially in a thin layer adjacent to the g.b. following a distribution function $h(r)$ with a maximum at $r=a-\lambda$, with $\lambda<a$ and $h(a)=h(a-2 \lambda)=0$. If at a given time there are $N$ gas atoms p.u. area of the g.b., the source term arising from resolution must be such that the volume integral of the distribution function verifies

$$
\int_{a-2 \lambda}^{a} h(r) 4 \pi I^{2} d r-4 \pi a^{2} \frac{b^{\prime} N}{2}
$$

where $b^{\prime}$ is the resolution rate of the g.b. bubbles. Thus, the gas generation rate term, $\beta$, of equation (4) is

$$
\beta- \begin{cases}y F & \text { for } 0 \leq r \leq a-2 \lambda, \\ y F+h(r) & \text { for } a-2 \lambda<r \leq a\end{cases}
$$

The equiaxed grain growth is expressed by the Ainscough (1973) model modified by Ito et al. (1985):

$$
\frac{d a}{d t}-k\left(\frac{1}{a}-\frac{f}{a_{m}}\right)
$$

where $k=3.64 \times 10^{-9} \exp (-63800 / R T)\left[\mathrm{m}^{2} \mathrm{~s}^{-1}\right], R=1.987 \mathrm{cal} \mathrm{mol}^{-1} \mathrm{~K}^{-1}$, $a_{\mathrm{m}}=1.1 \times 10^{-3} \exp (-7620 / \mathrm{T})[\mathrm{m}]$ and $f=1+v N / N_{\mathrm{s}} \cdot N_{\mathrm{s}}$, the number of gas atoms p.u. area that saturates the g.b., is

$$
N_{s}-\left(2 \gamma+x_{f} P_{\text {ext }}\right) \times \frac{4 w F_{s}}{3 k T}
$$

where $r_{f}$ is the bubble's curvature radius, $k=1.38 \times 10^{-23} \mathrm{~J} \mathrm{~K}^{-1}$, $F_{s}$ is the covered fraction of the grain faces, $\gamma$ is the surface stress of the bubbles, $w$ is a geometrical parameter and $P_{\text {ext }}$ is the external pressure exerted on the bubble. The temperature dependence of $N_{s}$ is such that it diminishes in two orders of magnitude from $\mathrm{T}=500 \mathrm{~K}$ to $\mathrm{T}=3000 \mathrm{~K}$.

\section{FISSION GAS RELEASE}

Before saturation, the gas content p.u. area of the g.b. at the time $t_{k}, N_{k}$, is determined by a balance equation which considers: $i$ ) the gas content of the grain and g.b. at the 
instant $k-1, i i)$ the amount of gas generated by fission during $\Delta t_{k}$ and $\left.i i i\right)$ the amount of gas incorporated to the grain and g.b. by sweeping of the g.b. Balance with the gas content of the grain and g.b. at $t_{k}$ gives

$$
C_{k-1}\left(\frac{a_{k}}{a_{k-1}}\right)^{3}+\frac{4}{3} \pi a_{k}^{3} y F \Delta t_{k}+4 \pi a_{k-1}^{2} \frac{N_{k-1}}{2}\left(\frac{a_{k}}{a_{k-1}}\right)^{3}-C_{k}+4 \pi a_{k}^{2} \frac{N_{k}}{2}
$$

with the initial conditions $(t=0, k=0) C_{0}=0$ and $N_{0}=0$. From here, we determine $N_{k}$ at every instant $t_{k}$ by means of

$$
\frac{4}{3} a_{k}^{3} \text { y } F t_{k}-C_{k}+4 \pi a_{k}^{2} \frac{N_{k}}{2}
$$

Once the saturation level is reached, the fission gases are released. The release at a given time $t_{k}$ is given by $R_{k}$

$$
R_{k}=\frac{4 \pi a_{k}^{2}}{2} \max \left[N_{k}-N_{s, k}, 0\right]
$$

where $4 \pi a_{k}{ }^{2} N_{k}$ represents all the atoms not contained within the grain. When saturation has not yet been achieved they are all allocated in the g.b. Otherwise, they are distributed between the g.b., $4 \pi a_{k}{ }^{2} N_{s}$, and the free volume of the fuel pin, $4 \pi a_{\mathrm{k}}^{2}\left(N_{\mathrm{k}}-N_{\mathrm{s}}\right)$.

The fractional release $F_{k}$ from the fuel with volume $v$, at the end of the $k$-th time step is given by

$$
F_{k}=\frac{R_{k}}{\frac{4}{3} \pi a_{k}^{3} y F t_{k}}
$$

This expression is valid both for one grain and for the whole fuel, since if $n_{k}$ grains are present, the generation and the release are multiplied by the same factor.

\section{RESULTS}

The diffusion equation (4) is solved by means of the implicit finite difference method (Denis et al. 1991). At $r=0$, due to symmetry, the condition $\partial \Psi / \partial r=0$ must be satisfied. At $r=a$, the condition of perfect sink of the g.b. implies $P=0$.

Figures 1-4 display our results (solid lines) together with those obtained by zimmermann (1978) (dashed lines) in isothermal experiments at $1250,1500,1750$ and $2000 \mathrm{~K}$. The parameters used in the calculations are listed in Table 1.

\section{CONCLUSIONS}

The calculation code is able to simulate numerically the grain growth, the amount of gas dissolved in the fuel matrix and accumulated in bubbles, either intra or interg., saturation of the latter and the consequent release of gas to the 
free volume of the fuel pin. The calculation time is sufficiently short as to make this code a good tool to be incorporated to other more general performance code.

Comparison between data reported in the literature of isothermal experiments and our calculations show a good agreement.

\section{Table 1}

$\Omega=4.09 \times 10^{-29}$
$\mu=10^{15}$
$Z=2$
$K=10^{5}$
$Y=0.3$
$\lambda=2 \times 10^{-8}$
$a=1 \times 10^{-5}$
$v=0.5$
$\gamma=0.626$
$r_{f}=5 \times 10^{-7}$
$P_{\text {ext }}=0$
$\theta=50$
$W^{\circ}=0.287$
$F_{s}=0.5$
$z_{0}=10^{-9}$
$I_{f}=6 \times 10^{-6}$
$\alpha=10$
$F=3.1 \times 10^{19}$
$\Delta X=\lambda / 4$
$b^{\prime}=1.55 \times 10^{-5}$

atomic volume sink strength of bubbles number of sites around a point defect rate of defect production per atom number of gas atoms created per fission event half-width of the re-entered atoms distribution initial grain radius parameter to calculate the maximum grain size surface stress of the bubbles radius of curvature of the lenticular bubbles external pressure on the g.b. bubbles half-angle between the surfaces of a g.b. bubble geometrical parameter of the g.b. bubbles fraction of g.b. surface occupied by bubbles range of influence of a fission fragment fission fragment range before coming to rest $\mathrm{n}$. of intrag. bubbles per fission fragment fission rate grid spacing used in the numerical calculation $b^{\prime}=1.55 \times 10^{-5}$ resolution rate of the grain boundary bubbles

The values of the parameters are in the SI units.

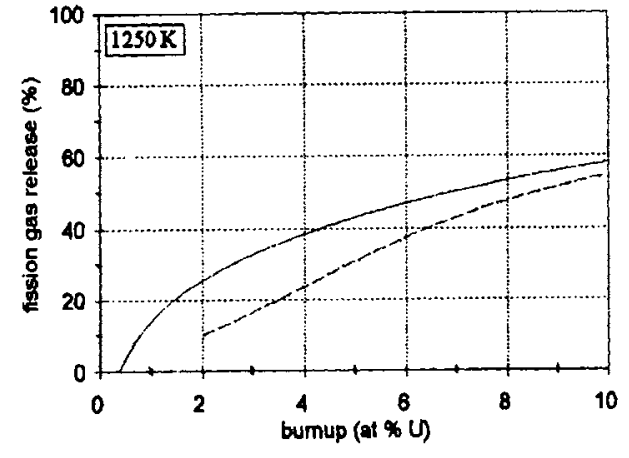

Figure 1

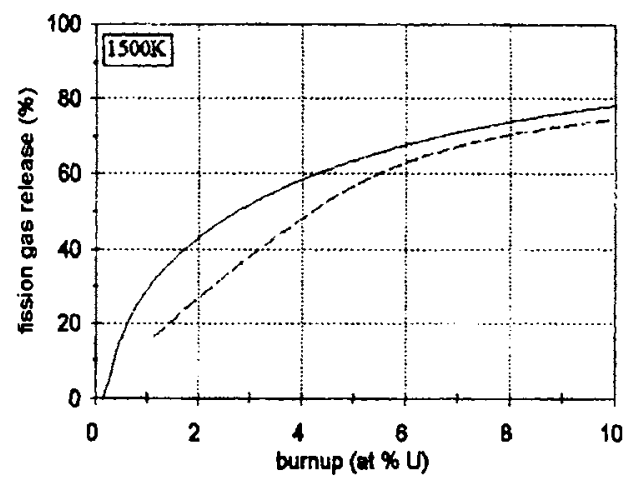

Figure 2 


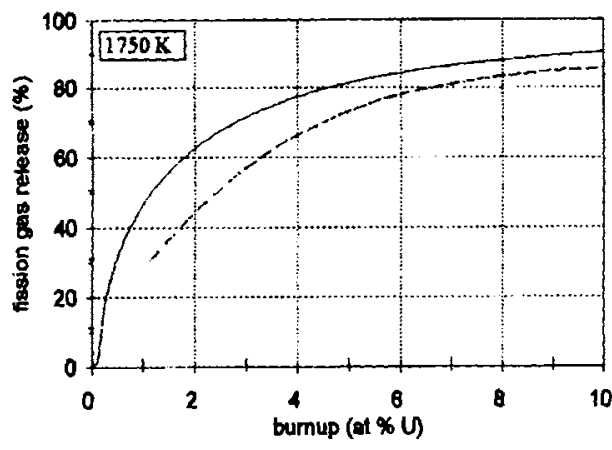

Figure 3

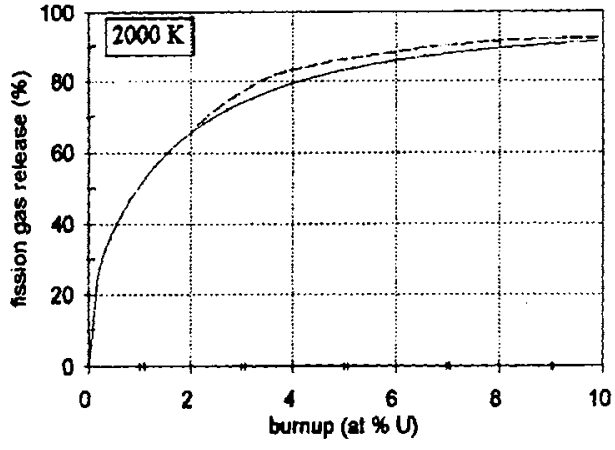

Figure 4

\section{REF ERENCES}

Ainscough, J., B.W. Oldfield \& J.O. Ware 1973/1974. Isothermal grain growth kinetics in sintered $\mathrm{UO}_{2}$ pellets. Journal of Nuclear Materials 49:117-128.

Denis A. \& E.A. Garcia 1991. Simulation with the HITO code of the interaction of zircaloy with $\mathrm{UO}_{2}$ and steam at high temperatures. Journal of Nuclear Materials 185:96-113.

Ito, K., R. Iwasaki \& Y. Iwano 1985. Finite element model for analysis of fission gas release from $\mathrm{UO}_{2}$ fuel. Journal of Nuclear Science and Technology 22[2]:129-138.

Nakajima, T. \& H. Saito 1987. A comparison between fission gas release data and FEMAXI-IV code calculations. Nuclear Engineering and Design 101:267-279.

Olander, D. 1976. Fundamental Aspects of Nuclear Reactor Fuel Elements. Berkeley: Technical Information Center, U.S. Department of Energy.

Turnbull, J., C. Friskeney, J. Findlay, F. Johnson \& A. Walter 1982. The diffusion coefficients of gaseous and volatile species during the irradiation of uranium dioxide. Journal of Nuclear Materials 107:168-184.

Turnbull, J., R. White \& C. Wise 1987. The diffusion coefficient for fission gas atoms in $\mathrm{UO}_{2}$. IAEA-TC-659/3.5:174181.

White, R. \& M. Tucker 1983. A new fission-gas release model. Journal of Nuclear Materials 118:1-38.

Zimmermann, H. 1978. Investigations on swelling and fission gas behaviour in $\mathrm{UO}_{2}$. Journal of Nuclear Materials 75:154161. 